\title{
Process and Clinical Outcomes of a Biosimilar Adoption Program with Infliximab-Dyyb
}

\author{
Shubha Bhat, PharmD, MS; Sarah Altajar, MD; Divya Shankar, MD; Toni Zahorian, PharmD; \\ Regine Robert, cPhT; Taha Qazi, MD; Bhavesh Shah, RPh; and Francis A. Farraye, MD, MSc
}

\begin{abstract}
BACKGROUND: In 2016, the FDA approved infliximab-dyyb (IFX-dyyb) as a biosimilar to infliximab (IFX). Deemed to have comparable efficacy and safety to IFX, IFX-dyyb is $20 \%-30 \%$ less expensive, allowing significant cost savings for institutions and some payers. In 2018, IFX was reported to be the drug with the highest spend since 2013 , costing $\$ 3.8$ billion; however, transition to IFX-dyyb would save $\$ 1.1$ billion. Regardless, many institutions have not transitioned to IFX-dyyb or other IFX biosimilars (e.g., IFXabda) because of concerns about clinical outcomes, uncertainty regarding financial impact, and barriers to operationalizing biosimilar adoption. At Boston Medical Center, a decision was made in March 2018 to adopt IFXdyyb and transition patients who have been on IFX for $\geq 6$ months for all indications to IFX-dyyb.
\end{abstract}

OBJECTIVES: To (a) describe a biosimilar adoption process of IFX-dyyb in patients on IFX for $\geq 6$ months; (b) characterize cost savings of transitioning patients to IFX-dyyb; and (c) evaluate real-world clinical outcomes of adult patients with inflammatory bowel disease (IBD) who transitioned to IFX-dyyb.

METHODS: This is a retrospective cohort study of patients eligible for the IFX-dyyb switch from March 2018 to June 2019 at a large academic medical center. For process outcomes, we collected the proportion of patients who transitioned to IFX-dyyb and calculated the cost savings generated. To assess clinical outcomes of adult IBD patients who transitioned, we collected IFX and IFX-dyyb dosage, Harvey Bradshaw Index (HBI) or Simple Clinical Colitis Activity Index (SCCAI) scores, c-reactive protein (CRP) levels, and colonoscopy results. Descriptive statistics, Wilcoxon signed-rank test, and McNemar's test were used for statistical analyses.

RESULTS: Of 151 eligible patients, 146 (97\%) successfully transitioned to IFX-dyyb. Based on our conversion rate to IFX-dyyb, our health system is forecasted to save approximately $\$ 500,000$ annually. From March to June 2018, 63 of 75 (84\%) eligible IBD patients transitioned from IFX to IFXdyyb. In this cohort, of the 40 patients with HBI or SCCAI scores before and after transition, $36(90 \%)$ maintained remission. For 32 patients, the mean CRP (SD) before transition was 11.2 (22) and 4.1 (4.8) after transition $(P=0.09)$. Since the IFX-dyyb transition, 9 patients had a colonoscopy, of which $5(56 \%)$ were in endoscopic remission. As of October 2018, $56(89 \%)$ patients continued with IFX-dyyb after transition. Of the 46 patients who had 12-15 months posttransition data, 38 (83\%) remained on IFX-dyyb.

CONCLUSIONS: Implementation of a biosimilar adoption program can be successful and result in significant cost savings without compromising clinical outcomes. A model that uses actionable strategies and embraces collaboration among stakeholders is described here, with outcomes demonstrating successful IFX-dyyb uptake and no changes in clinical outcomes of transitioned adult patients with IBD.

J Manag Care Spec Pharm. 2020;26(4):410-16

Copyright $\odot 2020$, Academy of Managed Care Pharmacy. All rights reserved.

\section{What is already known about this subject}

Given comparable efficacy and safety, biosimilars for infliximab have been approved by the FDA and can generate significant cost savings for payers and institutions; regardless, uptake has been slow because of clinical concerns, providers' inexperience with biosimilars, limitation in infrastructure to operationalize conversion, and lack of coverage or unfavorable reimbursement from payers.

Multiple stakeholders, including payers, providers, pharmacists, and patients, are influential in implementing a biosimilar adoption program.

Per clinical trials, infliximab biosimilars have shown to have comparable safety and efficacy compared with the originator product.

\section{What this study adds}

A model of how to successfully implement a biosimilar adoption program with actionable strategies is discussed, and a roadmap that can be applied to other biosimilar adoptions is provided. With appropriate stakeholder engagement, transitioning patients from infliximab to a biosimilar can be a successful endeavor and result in significant cost savings in a real-world setting.

This study reaffirms that the clinical outcomes of transitioning patients with inflammatory bowel disease from an originator product to an infliximab biosimilar should not be a barrier to biosimilar uptake.

I n 2016, the U.S. Food and Drug Administration (FDA) approved infliximab-dyyb (IFX-dyyb, Inflectra) as a biosimilar for infliximab (IFX, Remicade). IFX-dyyb met all of the FDA's biosimilarity requirements in that it is highly similar in structure and function, demonstrated comparative efficacy and safety, works through the same mechanism of action (tumor necrosis factor-a binding and neutralization), and is dosed identically to IFX. Subsequently, the FDA approved 3 additional IFX biosimilars: IFX-abda (Renflexis), IFX-qbtx (Ixifi), and infliximab-axxq (Avsola). Of note, Pfizer developed IFX-qbtx on its own before acquiring another company, Hospira, which had already developed and marketed IFX-dyyb in 2015, so IFX-qbtx was not launched in the United States. Given the recent approval of infliximab-axxq, it is not commercially available in the United States yet. In 2018, IFX was reported to be the drug with the highest spend since $2013 .{ }^{1}$ 
Biosimilars have the potential advantage of being significantly less expensive than originator biologics and therefore are of interest to institutions and some payers, given the inherent cost savings (estimated $\$ 1.1$ billion currently and $\$ 54-\$ 250$ billion over 10 years). ${ }^{1-3}$

Previous studies evaluating the transition from IFX originator products to biosimilars in patients with inflammatory bowel disease (IBD), rheumatoid arthritis, ankylosing spondylitis, psoriatic arthritis, and plaque psoriasis have found comparable efficacy and safety outcomes. ${ }^{4}$ The NOR-SWITCH trial, which assessed outcomes of patients who transitioned from IFX (after a minimum of 6 months) to IFX-dyyb versus patients who continued with IFX, did not find significant clinical differences across all indications. ${ }^{5}$ Specifically, in IBD patients who switched from IFX to IFX-dyyb, 82\% of patients continued treatment through 1 year and did not experience worsening of disease activity scores and inflammatory markers nor serious medication-related adverse effects. ${ }^{6}$

Regardless of existing clinical studies demonstrating comparable efficacy and safety, along with the cost reductions associated with infliximab biosimilars, a recent survey of pharmacy representatives from over 50 health systems shows that $62 \%$ of respondents have not yet transitioned. ${ }^{7}$ Another study found that IFX biosimilars only account for $2.4 \%$ of all IFX infusions in the United States. ${ }^{1}$ Adoption of biosimilars in the United States has been slow for numerous reasons, including prescriber and patient hesitation about clinical outcomes, payer restrictions, and lack of standardized operations. ${ }^{8}$ However, overcoming these barriers to biosimilar adoption could lead to increased patient access and major cost savings in health care.

The objectives of this study were to (a) describe a biosimilar adoption process for large-scale conversion to IFX-dyyb in patients on IFX for $\geq 6$ months for all indications; (b) highlight cost savings of transitioning patients to IFX-dyyb to health systems; and (c) evaluate clinical outcomes of adult patients with IBD who transitioned to IFX-dyyb at a large academic medical center.

\section{Methods}

\section{Biosimilar Adoption Process}

In the fall of 2017, pharmacy leadership proposed adopting IFX-dyyb for use at Boston Medical Center. During this time, stakeholders from the departments of dermatology, gastroenterology (adult and pediatric), and rheumatology were identified and invited to be a part of the biosimilar adoption process. Of these departments, only the gastroenterology outpatient practice had an embedded pharmacy team that consisted of an ambulatory care clinical pharmacy specialist and a certified pharmacy technician. This pharmacy team took the lead in the evaluation, education, and implementation of IFX-dyyb across the health system.
Initially, the pharmacy lead met with individual provider champions from each specialty to establish consensus and address clinical concerns before going to the formulary committee.

Providers across all specialties expressed the following concerns: appropriate timepoint for biosimilar switch, lack of knowledge about financial implications to patients converting to a biosimilar, and need for patient education/notification before the actual switch to address patient concerns and prevent interruptions in the infusion schedule and workflow. Gastroenterology providers were additionally concerned about indication extrapolation (e.g., biosimilar approved for indications not evaluated during the biosimilar's clinical trials) data, given the large number of patients currently on IFX.

To address these concerns, the pharmacy team (a) put together all clinical evidence from the United States and Europe; (b) consulted with European providers in the United Kingdom, Denmark, and Spain who were part of a European gastrointestinal network to discuss their clinical and operational process experiences with an IFX-dyyb switch; and (c) implemented close monitoring of clinical outcomes in adult patients with IBD per request of the gastroenterology faculty.

To address the patient education concern, the clinical pharmacist in gastroenterology created IFX-dyyb patient education materials for providers to hand out and for nurses in the infusion center to have available. In addition, the pharmacy team contacted each patient directly via phone to discuss the transition as soon as the IFX-dyyb prior authorization was approved and at least 2 weeks before the patient's first IFXdyyb infusion. Although literature exists regarding switching patients in Europe at various time points, providers felt more comfortable switching patients after 6 months of receiving IFX therapy, replicating the NOR-SWITCH trial. ${ }^{5}$ The pharmacy team also created talking points for providers regarding financial implications of switching to a biosimilar for patients and the health system.

Since IFX-dyyb does not carry an interchangeable designation, in which the biosimilar may automatically be used in place of the original product, members of the pharmacy department collaborated to create a monograph for IFX-dyyb to be presented at Boston Medical Center's pharmacy \& therapeutics (P\&T) committee meeting. The monograph included clinical parameters, product characteristics, projected annual cost, payer coverage and reimbursement analysis, and a failure mode analysis. The monograph also specified that patients eligible for the transition to IFX-dyyb must meet the following criteria: (a) be on IFX for $\geq 6$ months; (b) have the transition approved by the prescribing provider and the patient; (c) have IFX-dyyb covered by the patient's insurance company; and (d) receive the IFX-dyyb infusion at Boston Medical Center's outpatient infusion center only.

To avoid multiple switches, any patient who received IFX at multiple infusion centers, primarily students, were excluded 
from the transition. The monograph also outlined that (a) all patients new to infliximab therapy, pediatric or adult, managed inpatient or outpatient would receive IFX-dyyb and (b) patients on IFX admitted to our institution requiring emergent therapy were allowed to receive IFX until discharged to the outpatient clinic.

Ultimately, provider buy-in was obtained by involving key stakeholders throughout the process, including an invitation to attend the P\&T committee meeting, and prioritizing patient safety as seen in the inclusion/exclusion criteria. As a result, the P\&T committee approved the formulary change from IFX to IFX-dyyb without any recommended alterations.

Immediately following formulary approval in December 2017, providers initiated biosimilar conversations with their patients and informed the patients that a pharmacist would reach out with more information. Providers were not able to speak with all IFX patients in this manner, but it initiated the biosimilar conversation for the majority and prepared the patients for further discussion with the clinical pharmacy specialist at a later date. In addition to patient education, an in-service presentation was scheduled for the nursing team in the infusion unit, and patient education materials were supplied to prepare them for questions from patients regarding the upcoming transition to IFX-dyyb. Nurses were also encouraged to call the clinical pharmacy specialist in gastroenterology for in-person consultations with any patient receiving IFX who had questions about IFX-dyyb. Finally, Pfizer and our distributor were contacted to establish appropriate inventory at our wholesaler before going live with the IFX-dyyb switch.

To identify patients meeting the inclusion criteria for transition to IFX-dyyb, a report of all patients on IFX was generated through the electronic health record and converted to an Excel file (Microsoft, Redmond, WA). The original file contained patient name, medical record number, date of birth, telephone number, and date of next scheduled infusion. The IFX start date had to be added to the Excel file manually, since it could only be found in office notes or infusion plans and was needed to ensure that patients had been on IFX for $\geq 6$ months.

Beginning in February 2018, patients meeting inclusion criteria and scheduled to receive IFX in March 2018 were targeted for transition to IFX-dyyb. Approximately 4 weeks before scheduled IFX infusion, the certified pharmacy technician in the gastroenterology clinic contacted the patient's payer to request approval for IFX-dyyb. The prior authorization process was initiated before contacting the patient or changing the prescription to ensure that IFX-dyyb would be covered. The majority of payers agreed to approve IFX-dyyb. Within a few months of continuous contact with payers, the prior authorization process became seamless. Also, beginning in March 2018, all patients new to IFX therapy started with IFX-dyyb, unless their payers did not cover it.

\section{TABLE 1 Outline of Information Included in} Patient Call

Introduction

- "My name is [insert name here]. I am a pharmacist at Boston Medical Center, and I work in the gastroenterology office with [insert provider name]"

Provide reason for call

- "I am calling to discuss a change that Boston Medical Center has made, after discussion with your provider, to change our preferred medication from Remicade (IFX) to a biosimilar, Inflectra (IFX-dyyb)"

- One of the benefits to using biosimilars is that they lower costs, which over time can improve insurance premiums, medication out-of-pocket costs, and medication access

- Assess patient's understanding of biosimilars, as he or she may have received medication information during his or her last visit to the office or the infusion center

- Ask patient if he or she has time for a discussion and would like to know more about biosimilars (caller may need to be corrected or further educated to address knowledge gaps)

Provide education on biosimilars

- Inflectra (IFX-dyyb) is a biosimilar, designed to be highly similar to the existing originator drug Remicade (IFX), and is FDA-approved to treat [insert patient condition]

- Although these medications are not identical, they work in the same way and have the same dose, administration (intravenous infusion), and side effects

- As with any medication, recommend the patient call his or her provider if starting any new medications or experiencing any unusual adverse effects

Explain how this change will affect the patient

- Confirm that medication is covered by insurance plan

- Reinforce the importance of adherence to infusion appointments

- Confirm date and time of next infusion

- Inform patient that infusion time and frequency will be unchanged from when they received IFX

Offer opportunity for patient to ask questions

- Provide call back number should patient think of any questions prior to scheduled infusion

- Contact provider if patient requests to speak with them directly and provide a timeline for follow-up with the patient to ensure their questions have been answered

\section{Offer additional resources}

- Online resources: Crohn's and Colitis Foundation and American Gastroenterological Association

FDA = U.S. Food and Drug Administration; IFX = infliximab.

Once prior authorization approval was obtained, the clinical pharmacy specialist in gastroenterology contacted all patients who would be transitioning to IFX-dyyb (for any indication) via phone to discuss the formulary change and answer any questions that these patients had regarding their treatment. The first call attempt was made approximately 2-3 weeks before the date of the first IFX-dyyb infusion to allow for multiple discussions and inclusion of the provider if requested by the patient. Patients could refuse the transition to IFX-dyyb, but this was rare given the advanced patient notice, individual education provided, and provider buy-in to the process. If a 


\begin{tabular}{|c|c|c|}
\hline & $\begin{array}{l}\text { Patients Who } \\
\text { Transitioned } \\
\text { to IFX-dyyb } \\
(\mathrm{n}=63)\end{array}$ & $\begin{array}{c}\text { Patients Who } \\
\text { Remained } \\
\text { on IFX } \\
(n=12)\end{array}$ \\
\hline Age, year (SD) & $38(13)$ & $34(12)$ \\
\hline Female, n (\%) & $21(33)$ & $6(50)$ \\
\hline \multicolumn{3}{|l|}{ Race, n (\%) } \\
\hline African American & $16(25)$ & $3(25)$ \\
\hline Caucasian & $42(67)$ & $8(67)$ \\
\hline Asian/other & $5 \quad(8)$ & $1(8)$ \\
\hline Hispanic & $7(11)$ & $1(8)$ \\
\hline IBD duration, year (SD) & $11 \quad(9)$ & $11(9)$ \\
\hline \multicolumn{3}{|l|}{ IBD type, n (\%) } \\
\hline Crohn disease & $41(65)$ & $10(83)$ \\
\hline Ulcerative colitis & $22(35)$ & $2(17)$ \\
\hline On immunomodulator therapy, n (\%) & $34(54)$ & $4(33)$ \\
\hline \multicolumn{3}{|l|}{ Before transition } \\
\hline Dose, mg/kg (range) & $7(5-10)$ & $7(5-11)$ \\
\hline Interval, week (range) & $7(4-8)$ & $6(4-8)$ \\
\hline IFX duration before transition, month (SD) & $31 \quad(15)$ & $28(14)$ \\
\hline \multicolumn{3}{|l|}{ After transition } \\
\hline Dose, mg/kg (range) & $7.6(5-11)$ & $7.8(5-11)$ \\
\hline Interval, week (range) & $6.8(4-8)$ & $6.6(4-8)$ \\
\hline
\end{tabular}

IBD = inflammatory bowel disease; IFX = infliximab; SD = standard deviation

patient could not be reached after 2 call attempts or did not have a working phone number on file, a letter was sent to the last known address, explaining the transition and providing a call back number for questions. Patient calls were semistructured so that the patients' questions could drive the discussion, but generally included open-ended questions to explore patients' knowledge of biosimilars possibly garnered from previous meetings with their providers, how it would affect their appointments, and an offer to answer any questions (Table 1). Patients were also directed to online resources with information about biosimilars and to relevant disease societies with guidance on the matter (e.g., IBD patients were referred to Crohn's and Colitis Foundation and the American Gastroenterological Association).

Once the patient provided consent to the IFX-dyyb switch, the provider was notified to update the prescription and infusion plan. The Excel file, maintained by the pharmacy team in gastroenterology, was used to track prior authorization, patient call outreach, and prescription/infusion plan update status.

\section{Data Collection and Measured Outcomes}

A retrospective study of all patients treated in the dermatology, gastroenterology, or rheumatology clinics at Boston Medical Center who transitioned from IFX (after being on this medication for $\geq 6$ months) to IFX-dyyb from March 2018 to June 2019 was performed. The proportion of patients who transitioned to IFX-dyyb was collected from the Excel file used to track the transition process in the office. A patient was considered to have successfully switched if the prescription/infusion plan was updated to IFX-dyyb. Reasons for nonconversions were also collected. To calculate cost savings, current and forecasted use of IFX vials, as determined by an internal analysis, and contracted institution's price for IFX-dyyb were used.

Given the agreement with gastroenterology providers to implement close monitoring of clinical outcomes in adult patients with IBD, clinical outcomes were assessed in this cohort of patients who were eligible to transition to IFX-dyyb from March to June 2018. This time frame was selected to ensure that patients had a minimum of 3 months of data after the IFX-dyyb transition at the time this analysis was conducted. Patients who switched to IFX-dyyb and those who continued on IFX were included. Patient demographics collected included age, gender, race, ethnicity, and type and duration of IBD. We collected the following variables before and after transition: IFX dose and interval (originator and biologic product), presence of concurrent immunomodulator use, Harvey Bradshaw Index (HBI) scores for Crohn disease, Simple Clinical Colitis Activity Index (SCCAI) scores for ulcerative colitis, c-reactive protein (CRP) levels, fecal calprotectin levels, and colonoscopy results.

The transition date was defined as the first day that a patient received IFX-dyyb. For patients who continued on IFX, the transition date was defined as March 5, 2018 (the first day patients could transition to IFX-dyyb). The most recent data before the transition date was collected for pretransition data. Posttransition data was collected at 3 months after transition for analyses purposes. If patients had data on IFX-dyyb beyond 3 months, additional data was collected for descriptive purposes. Outcome measures included proportion of patients who transitioned to IFX-dyyb and clinical outcomes in the posttransition phase

Descriptive statistics, Wilcoxon signed-rank test, and McNemar's test were used for statistical analyses. SAS version 9.4 was used (SAS Institute, Cary, NC). This study was approved by the Boston Medical Center Institutional Review Board.

\section{Results}

From March 2018 to June 2019, 179 patients were identified as receiving IFX for $\geq 6$ months and eligible to transition to IFXdyyb. Within this cohort, 15 (8\%) patients were not eligible to switch because of payer denial; 7 (4\%) patients received some of their IFX infusion at other centers; 3 (2\%) patients were not deemed to be on a stable IFX regimen by their providers; and 3 (2\%) refused to transition. Thus, 151 patients met all the criteria for IFX-dyyb transition. In this population, 146 (97\%) 
successfully transitioned. The remaining 5 patients did not transition because IFX was discontinued for another treatment option ( $n=3,2 \%$ ); they transferred care for reasons not related to the IFX-dyyb switch $(n=1,1 \%)$; or were lost to follow-up ( $n=1$, $1 \%$ ). The majority of patients who successfully transitioned were managed by the pediatric or adult gastroenterology practice. Patients were on IFX for an average of 27 (range 6-49) months before transitioning to IFX-dyyb. Based on the proportion of patients we converted, the calculated cost savings for our health system was forecasted to be $\$ 500,000$ annually.

In our clinical outcomes analysis of patients with IBD between March and June 2018, 63 of 75 (84\%) eligible patients transitioned from IFX to IFX-dyyb; however, 12 remained on IFX because of insurance restrictions. Table 2 lists patients' demographics. Per HBI and SCCAI scores available before and after transition for 40 patients, 36 (90\%) maintained remission; $2(5 \%)$ not in remission on IFX attained remission on IFXdyyb; and 1 (2\%) in remission on IFX reported an increased HBI score on IFX-dyyb, although the colonoscopy showed no active Crohn disease. Per CRP data available for 32 individuals, mean CRP (standard deviation [SD]) before transition was 11.2 $( \pm 22)$ vs. $4.1( \pm 4.8)$ after transition $(P=0.09)$. After IFX-dyyb transition, 9 patients had colonoscopies, of which 5 (56\%) showed endoscopic remission. As of October 2018, 32 (51\%) patients continued with the current IFX-dyyb dose, while $24(38 \%)$ required a dose increase. Four (6\%) patients discontinued IFX-dyyb because of antibody development $(n=2)$ or worsening disease $(n=2)$, and $3(5 \%)$ transferred care or were lost to follow-up. Conversely, of the 12 patients on IFX, $9(75 \%)$ continued on the same dose; 2 (17\%) required a dose increase; and 1 (8\%) transferred care. In the IFX-dyyb cohort, 46 patients had data $12-15$ months after transition, in which the majority $(n=38,83 \%)$ remained on IFX-dyyb.

\section{Discussion}

This study is unique in describing a successful biosimilar adoption program with IFX-dyyb in a large academic medical center. Of patients who met all the criteria, the rate of patients converted from IFX to IFX-dyyb was $97 \%$, with an estimated cost savings of $\$ 500,000$ annually. Furthermore, no significant clinical differences were noted in adult patients with IBD who transitioned from IFX to IFX-dyyb. These findings reinforce that certain barriers to biosimilar uptake can be successfully addressed to implement a robust biosimilar adoption program.

Barriers affecting biosimilar uptake have been previously documented and discussed in the literature. ${ }^{4,8-13}$ These studies highlight that biosimilar adoption is heavily influenced by stakeholders, including providers, patients, and payers. ${ }^{14,15}$
From a payer perspective, decision making is often influenced by the cost difference between a biosimilar and its reference product or rebates that they may receive from the manufacturers for formulary preference. ${ }^{16}$ Alternatively, providers base their prescribing of medications on efficacy and safety data. ${ }^{10}$ From a patient perspective, "a biosimilar" is often a foreign term or considered to be inferior to the reference product. ${ }^{11}$ A recent survey disseminated to 300 managed care and specialty pharmacy professionals found that one third of respondents endorsed formulary preferences for biosimilars being driven by contracting rebates; alternatively, $25 \%$ of respondents stated biosimilar adoption had not occurred at their organization because of safety and efficacy concerns. ${ }^{15}$ These findings reiterate that biosimilar barriers are likely universal and align with perceptions that we had to address to successfully implement the biosimilar adoption program.

The actionable strategies used in the implementation and acceptance of the biosimilar adoption program included (a) collaboration among multiple stakeholders for buy-in and structured implementation process and (b) education of biosimilar efficacy and safety to providers and patients. Our targeted approach was supported by a recently published study in which prescriber education programs-which incorporate evidenced-based findings and postmarketing information to highlight real-world outcomes-and partnership between stakeholders were identified as the most effective approaches to overcome biosimilar adoption barriers. ${ }^{15}$ Identifying a champion provider and having the pharmacy department align all stakeholders involved in the process was critical for successful biosimilar conversion. Furthermore, given that the pharmacy team plays multiple roles within the institution (part of the P\&T process, overseeing prior authorizations, and providing medication-related education), the clinical pharmacist and pharmacy technician were well situated to lead the implementation of the biosimilar adoption program. In this process, payer acceptance of our formulary change still presents as the biggest barrier. Future advocacy efforts in regard to rebate contracts and interchangeability status may lead payers to be more accepting of biosimilar transition. ${ }^{17}$

One other actionable strategy to improve biosimilar uptake is to monitor real-world clinical outcomes after biosimilar transition. ${ }^{15}$ In addition to reviewing efficacy and safety data with providers and patients, providing real-world outcome data can be more powerful than randomized controlled studies. Our assessment of the clinical outcomes of adult patients with IBD who transitioned to an IFX biosimilar showed that the majority of patients remained in remission and continued to be treated with IFX-dyyb 1 year after transition.

Our findings align well with other postmarketing studies, which found that switching from the IFX originator to 
a biosimilar did not influence the maintenance of clinical remissions or result in more adverse events. ${ }^{18-21}$ Another study, which included 100 patients with IBD, assessed outcomes of patients who transitioned to an IFX biosimilar for 2 years and found that $72 \%$ of patients stayed on the biosimilar; $69.9 \%$ and $68.5 \%$ attained remission at 18 and 24 months; and no significant changes in HBI, partial Mayo score, and CRP were noted. ${ }^{22}$ These data are reassuring, and thus, institutions looking to implement a successful biosimilar adoption program should also include an internal assessment of patient outcomes after the transition, as well as a review of real-world data with stakeholders in a timely manner. ${ }^{15}$

\section{Limitations}

This study has some limitations to consider. The assessment of clinical outcomes was limited to adult patients with IBD who transitioned from IFX to IFX-dyyb over a short time frame, which generated a small sample size for evaluation. As previously mentioned, the time frame was chosen to ensure that patients had at least 3 months of IFX-dyyb data for follow-up and to internally assess outcomes after transition to maintain provider and patient buy-in. Ongoing clinical assessment of adult patients with IBD, as well as pediatric patients and those with other indications who transition to IFX-dyyb, are warranted.

Also, our study only addresses 1 model for implementing a biosimilar adoption program, which may not be applicable to other institutions, particularly those without a clinical pharmacist and certified pharmacy technician embedded within the clinic. These institutions may be able to tap into other resources, such as having the specialty pharmacy team take the lead. Regardless of the model implemented, engagement with all stakeholders is the key to successful implementation efforts. Future studies assessing other models for successful implementation of a biosimilar adoption program are warranted.

\section{Conclusions}

Transitioning patients from IFX to IFX-dyyb can be operationalized at a large scale with $97 \%$ conversion rate, which can translate to significant cost savings as long as collaborative efforts between stakeholders are maintained. Payers may present a significant barrier, but emerging real-world data, increase in payer communication, and future advocacy efforts could bridge that gap. In our assessment of clinical outcomes within the IBD cohort, we found that a high proportion of patients transitioned and remained on IFX-dyyb. Also, no clinically significant differences in outcomes were seen. The strategies and model used in this program provide a robust biosimilar adoption road map that can be executed at other institutions to increase patient access to biosimilar therapies and generate significant cost savings to the health care system.

\section{Authors}

SHUBHA BHAT, PharmD, MS; TONI ZAHORIAN, PharmD; REGINE ROBERT, cPhT; and BHAVESH SHAH, RPh, Department of Pharmacy, Boston Medical Center, Boston, Massachusetts. SARAH ALTAJAR, MD, and DIVYA SHANKAR, MD, Department of Medicine, Boston Medical Center, Boston, Massachusetts. TAHA QAZI, MD, Department of Gastroenterology, Boston Medical Center, Boston, Massachusetts, and FRANCIS A. FARRAYE, MD, MSc, Inflammatory Bowel Disease Center, Division of Gastroenterology and Hepatology, Mayo Clinic, Jacksonville, Florida.

AUTHOR CORRESPONDENCE: Shubha Bhat, PharmD, MS, Department of Pharmacy, Boston Medical Center, 725 Albany St., Ste. 6C, Boston, MA 02118. Tel.: 617.638.5919;

E-mail: shubha.bhat@bmc.org.

\section{DISCLOSURES}

No outside funding supported this study. Farraye reports advisory board fees from Janssen, Merck, and Pfizer. Shah reports speaker fees from Pfizer. The other authors have nothing to disclose.

\section{REFERENCES}

1. Schumock GT, Stubbings J, Wiest MD, et al. National trends in prescription drug expenditures and projections for 2018. Am J Health Syst Pharm. 2018;75(14):1023-38

2. Kaida-Yip F, Deshpande K, Saran T, Vyas D. Biosimilars: review of current applications, obstacles, and their future in medicine. World J Clin Cases. 2018;6(8):161-16.

3. Mulcahy AW, Hlavka JP, Case SP. Biosimilar cost savings in the United States: initial experience and future potential. Rand Health Q. 2018;7(4):3.

4. Feagan BG, Lam G, Ma C, Lichtenstein GR. Systematic review: efficacy and safety of switching patients between reference and biosimilar infliximab. Ailment Pharamcol Ther. 2019;49(1):31-40.

5. Jorgensen KK, Olsen IC, Goll GL, et al. Switching from originator infliximab to biosimilar CT-P13 compared with maintained treatment with originator infliximab (NOR-SWITCH): a 52-week, randomized, double-blind, non-inferiority trial. Lancet. 2017;389(10086):2304-16.

6. Smits LJT, Grelack A, Derikx LAAP, et al. Long-term clinical outcomes after switching from Remicade to Biosimilar CT-P13 in inflammatory bowel disease. Dig Dis Sci. 2017;62(11):3117-22.

7. Oskouei ST. Following the biosimilar breadcrumbs: when health systems and manufacturers approach forks in the road. J Manag Care Spec Pharm. 2017;23:1245-48. Available at: https://www.jmcp.org/doi/10.18553/ jmcp.2017.23.12.1245.

8. Hakim A, Ross JS. Obstacles to the adoption of biosimilars for chronic diseases. JAMA. 2017;317(21):2163-64.

9. Leonard E, Wascovich M, Oskouei S, Gurz P, Carpenter D. Factors affecting health care provider knowledge and acceptance of biosimilar medicines: a systematic review. J Manag Care Spec Pharm. 2019;25(1):102-12. Available at: https://www.jmcp.org/doi/10.18553/jmcp.2019.25.1.102.

10. Cohen H, Beydoun D, Chen D, et al. Awareness, knowledge, and perceptions of biosimilars among specialty pharmacy physicians. Adv Ther. 2017;33(12):2160-72

11. Jacobs I, Singh E, Sewell KL, Al-Sabbagh A, Shane LG. Patient attitudes and understanding about biosimilars: an international cross-sectional survey. Patient Prefer Adherence. 2016;10:937-48. 
12. Bonovas S, Peyrin-Biroulet L, Danese S. Clinical development of biologicals and biosimilars-safety concerns. Expert Rev Clin Pharmacol. 2017;10(6):567-69.

13. Boccia R, Jacobs I, Popovian R, de Lima Lopes G Jr. Can biosimilars help achieve the goals of the US health care reform? Cancer Manag Res. 2017;9:197-205

14. Crepsi-Lofton J, Skelton JB. The growing role of biologics and biosimilars in the United States: perspectives from the APhA Biologics and Biosimilars Stakeholder Conference. J Am Pharm Assoc (2003). 2017;57(5):e15-27.

15. Greene L, Singh RM, Carden MJ, Pardo CO, Lichtenstein GR. Strategies for overcoming barriers to adopting biosimilars and achieving goals of the Biologics Price Competition and Innovation Act: a survey of managed care and specialty pharmacy professionals. J Manag Care Spec Pharm. 2019;25(8):904-12.

16. Kim Ws, Ogura M, Kwon HC, Choi D. Looking to the future and learning lessons from the recent past: changing stakeholder perceptions of biosimilars in cancer. Future Oncol. 2017;13(15s):17-29.

17. Smeeding J, Malone DC, Ramchandani M, et al. Biosimilars: considerations for payers. P T. 2019;44(2):54-63.
18. Guiotto C, Italia A, Lavagna A, et al. Switching from infliximab originator to a first biosimilar is safe and effective. Results of a case-control study with drug levels and antibodies evaluation. Dig Liver Dis. 2019;51(8):1117-22.

19. Ye BD, Pesegova M, Alexeeva O, et al. Efficacy and safety of biosimilar CT-P13 compared with originator infliximab in patients with active Crohn's disease: an international, randomised, double-blind, phase 3 non-inferiority study. Lancet. 2019;393(10182):1699-707.

20. Gheorghe C, Svoboda P, Mateescu B. Effectiveness and safety of biosimilar infliximab (CT-P13) in a real-life setting in patients with Crohn's disease or ulcerative colitis. J Drug Assess. 2019;18(1):129-34.

21. Tursi A, Mocci G, Faggiani R, et al. Infliximab biosimilar CT-P13 is effective and safe in treating inflammatory bowel diseases: a real-life multicenter, observational study in Italian primary inflammatory bowel disease centers. Ann Gastroenterol. 2019;32(4):392-99.

22. Guerra Veloz MF, Belvis Jimenez M, Valdes DT, et al. Long-term follow up after switching from original infliximab to an infliximab biosimilar: realworld data. Therap Adv Gastroenterol. 2019;12:1756284819858052. 